\title{
Pattern and intensity of physical activity
}

\section{Keeping moderately active is the best way to boost total daily energy expenditure.}

A low level of physical activity is a pervasive feature of our modern lifestyle. Attempts to counteract the negative effects of sedentary living include introducing bouts of high-intensity exercise, but I show here that it can be more effective to increase the amount of time spent on activities of moderate intensity while reducing periods of inactivity during waking hours.

Physical inactivity may be associated not only with being overweight but also with disorders such as coronary heart disease or type-2 diabetes ${ }^{1}$, which may be prevented by exercising regularly ${ }^{2}$. Consequently, sedentary people often include spells of vigorous exercise in an otherwise inactive and unhealthy lifestyle. I have tested whether this is the most effective way to enhance people's total daily activity levels by determining how these are influenced by exercise duration and intensity.

Healthy, non-obese adults (14 women and 16 men; $27 \pm 5 \mathrm{yr} ; 24.1 \pm 2.3 \mathrm{~kg} \mathrm{~m}^{-2}$ ) participated in the study ${ }^{3}$. Total energy expenditure was measured by monitoring their metabolism with doubly labelled water over a two-week period, the optimal observation interval for the biological half-lives of the isotopes. The physical activity level (PAL) is defined as the factor by which total energy expenditure exceeds resting energy expenditure, measured during an overnight stay in a respiration chamber.

To determine the amount of body movement over 1-min intervals, I recorded movements over the first of the two weeks with a 'Tracmor' portable motion sensor. This consists of a body-fixed triaxial accelerometer and a data unit for on-line registration, processing and storage of acceleration signals. The triaxial accelerometer $(50 \times 30 \times 8 \mathrm{~mm} ; 16 \mathrm{~g})$ consists of three uniaxial piezoresistive accelerometers and registers accelerations in three orthogonal directions as the body moves. Acceleration signals are processed to obtain the sum of the rectified and integrated acceleration curves from all three measurement directions. The integration time is set at $1 \mathrm{~min}$ and the final output is expressed as counts per minute (c.p.m.) $)^{4}$.

Using data from Tracmor recordings in combination with activity details recorded in diaries, three activity categories were defined: low intensity (lying, sitting and standing; Tracmor output under 1,000 c.p.m.), moderate intensity (walking and cycling; output 1,000-3,000 c.p.m.); and high intensity (housework, gymnastics and sport; output greater than 3,000 c.p.m.).

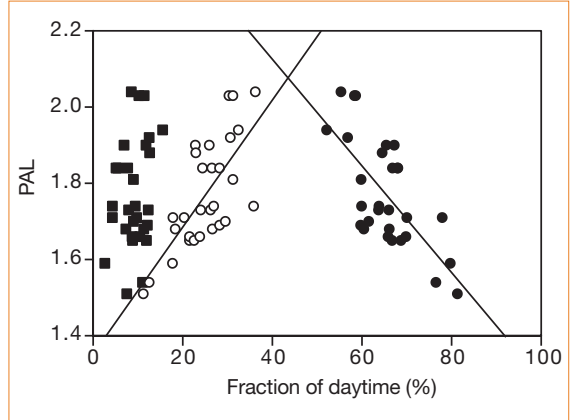

Figure 1 Physical activity levels (PAL) as a function of the fraction of daytime hours spent by 30 healthy subjects (with body-mass indices within the normal range) on activities of low (filled circles), moderate (circles) and high (squares) exercise intensity. Linear regressions are shown for low- and moderate-intensity activities: subjects spending more time on moderate-intensity exercise and less on low-intensity activity can improve their PAL values. Time spent on high-intensity exercise does not appear to influence total energy expenditure.

Calculated PAL values fell in the range 1.51-2.04 for all subjects, apart from one woman whose PAL value was 2.57; her data were excluded as an outlier. Three subjects reported not wearing the Tracmor while performing intensive sports activities, so the time spent on high-intensity activity by these subjects was corrected accordingly.

In a multiple regression analysis with the fraction of time spent on activities of moderate and high intensity, only moderateintensity activity came out as a significant predictor of PAL $\left(r^{2}=0.51, P<0.0001\right)$. Subjects spending relatively more time on moderate-intensity activity and therefore less on low-intensity activity had a higher PAL value (Fig. 1). There was, however, no relation between PAL value and the time spent on just high-intensity activity, presumably because this was limited by its nature to being relatively short.

In the general population, PAL ranges between 1.2 and 2.2-2.5. At PAL values of about 2.5 , subjects have problems main-

\section{Forensic palaeontology}

\section{The Archaeoraptor forgery}

he Archaeoraptor fossil was announced as a 'missing link' and purported to be possibly the best evidence since Archaeopteryx that birds did, in fact, evolve from certain types of carnivorous dinosaur ${ }^{1}$. It reportedly came from Early Cretaceous beds of China that have produced other spectacular fossils transitional taining energy balance and may lose weight; PAL is about 1.5 or 2.1 for sedentary or very active people, respectively ${ }^{5}$. My results indicating that short periods of vigorous activity do not have much impact on PAL in the normal population are borne out by studies on obese patients: adding exercise to an energy-restricted diet does not further increase weight loss ${ }^{6,7}$ because the costs of the extra activity are compensated by a reduction of energy spent on physical activity outside the training sessions ${ }^{8}$.

My results show, however, that the proportion of time distributed between activities of low and moderate intensity is what influences the total energy expenditure and so determines the value of PAL. Subjects wanting to increase their metabolic rate should exchange low-intensity activities such as sitting in front of a screen for moderate-intensity activities such as walking or cycling. Moderate-intensity activities are better tolerated than high-intensity activities, especially by the middle-aged or obese. An improvement in overall activity levels should bring important health benefits as subjects expend more energy and reduce their risk of running into positive energy balance and gaining weight.

Klaas R. Westerterp

Department of Human Biology, Maastricht

University, 6200 MD Maastricht, The Netherlands e-mail:k.westerterp@hb.unimaas.nl

1. Booth, F. W., Gordon, S. E., Carlson, C. J. \& Hamilton, M. T. J. Appl. Physiol. 88, 774-787 (2000).

2. Grundy, S. M. et al. Med. Sci. Sports Exerc. 31 (suppl.), 502-508 (1999).

3. Westerterp, K. R. et al. Br. J. Nutr. 76, 785-795 (1996).

4. Bouten, C. V. C., Verboeket-van de Venne, W. P. H. G.,

Westerterp, K. R., Verduin, M. \& Janssen, J. D. J. Appl. Physiol. 81, 1019-1026 (1996).

5. Westerterp, K. R.. Am. J. Clin. Nutr. 68 (suppl.), 970-974 (1998).

6. Ballor, D. L. \& Poehlman, E. T. Eur. J. Appl. Physiol. 71, 535-542 (1995).

7. Garrow, J. S. \& Summerbell, C. D. Eur. J. Clin. Nutr. 49, 1-10 (1995).

8. Kempen, K. P. G., Saris, W. H. M. \& Westerterp, K. R. Am. J. Clin. Nutr. 62, 722-729 (1995)

between birds and extinct non-avian dinosaurs ${ }^{2,3}$. But Archaeoraptor was revealed to be a forgery in which bones of a primitive bird and a non-flying dromaeosaurid dinosaur had been combined ${ }^{4-6}$. Here we use high-resolution X-ray computed tomography $(\mathrm{CT})^{7}$ to determine the nature and extent of the forgery, as well as how it was built, by imaging the fracture pattern and distribution of materials through the entire specimen.

The Archaeoraptor specimen, which was reportedly collected from the Early Creta- 
ceous Jiufotang Formation of Liaoning, was smuggled out of China and later sold in the United States on the commercial market ${ }^{4}$. Before repatriation ${ }^{5}$, the specimen, which was alleged to contain a single complete skeleton, was brought to us for CT analysis.

The slab had obviously been reassembled from numerous broken pieces, and the skeleton presented a unique combination of features, including teeth, feathers, a more flight-capable wing than Archaeopteryx, and the tail of a non-flying dromaeosaur. The entire bone-bearing portion of the slab was scanned in 422 consecutive 1-mm-thick slices, spaced at $0.9 \mathrm{~mm}$ (for the full dataset and description of scanning protocols, see supplementary information).

Computed tomography alone cannot distinguish genuine and fraudulent mistakes, but it provides criteria for evaluating whether pairs of adjacent pieces lie in natural relation to one another. These include comparison of adjacent fracture-face geometries, thickness and density of adjacent pieces, cross-cutting relationships among four generations of fractures, the distribution of grout, and continuity across fractures of bones, natural moulds of bones, and invertebrate burrows. The textural signature of the grout is its relatively low density, inclusion of air bubbles and metallic particles, a unique fracture pattern, and dark green fluorescence under ultraviolet light. Overlapping relationships among blobs of grout and different pieces, which can be observed in CT cross-section, reveal the general sequence of assembly.

The Archaeoraptor slab was built in three layers. The top 'layer' is a heterogeneous mosaic of 88 separate pieces, some of which contain bone (Fig. 1). The bottom layer is a single, unbroken plate of shale used for backing. Between the two is a layer of grout, which was spread over the face of the backing plate and between ruptured edges of the top pieces, as they were set into place. Many Liaoning fossils have undergone this same style of repair.

The top layer was built in three phases. The first to be assembled were 23 pieces that lie together in natural relationships and contain the anterior half of an articulated bird skeleton (to be described as a new species; X. Xu et al., manuscript in preparation). In the next phase, 26 pieces containing bones and a few contiguous pieces were sequentially articulated against the rear half of the skeleton to 'complete' it. None of these pieces preserves evidence of a natural attachment to the bird skeleton pieces, and in only a few cases are these pieces naturally associated with one another. Also, the 'paired' tibiae/fibulae and feet are split parts and counterparts from a single side, positioned as if they were right and left.

The third phase of construction involved placement of 39 'shims'. The shims lack
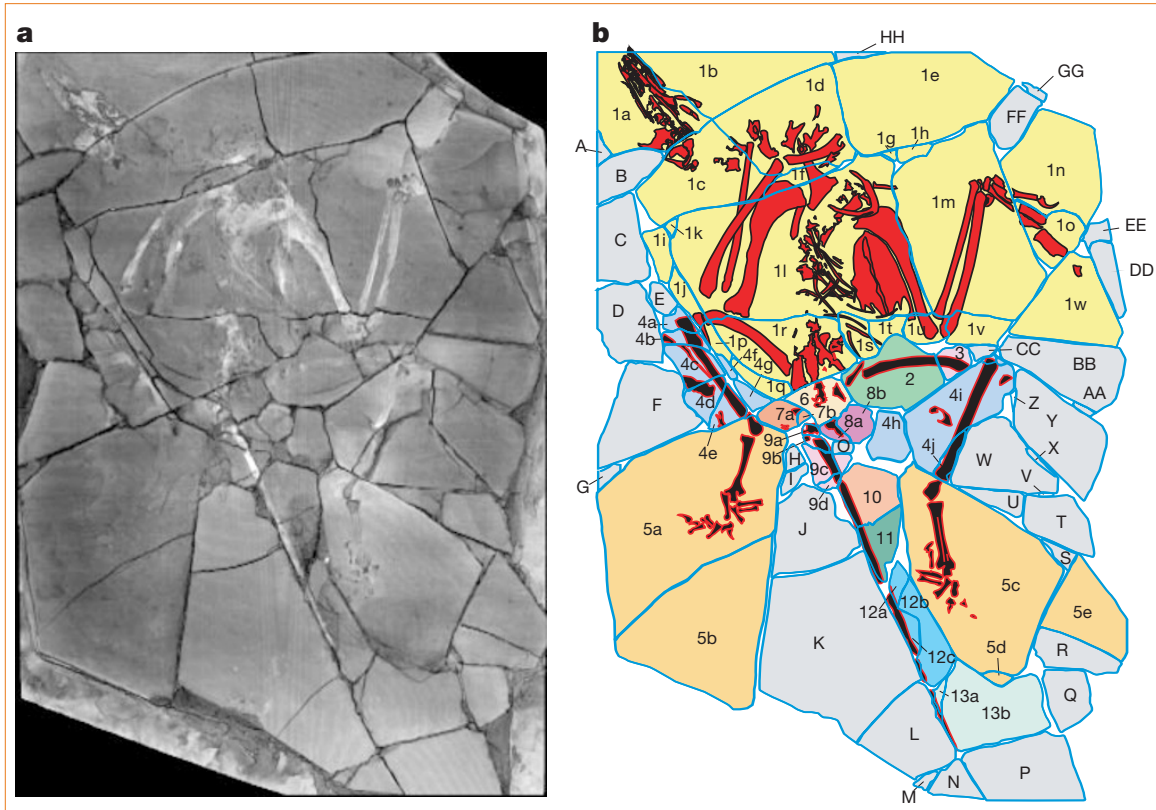

Figure 1 Two computer-generated models of the face of the Archaeoraptor slab as it was presented for computed tomography (CT) scanning on 29 July 1999. The specimen was scanned from top to bottom in planes perpendicular to this view. a, Volumetric model generated from superimposed CT slices. Light grey, bones; dark grey, slab materials; black, air. b, Map of the slab face, colour-coded to indicate the nature of the associations between its 88 constituent pieces. Red, associated bird bones; black, unverifiable 'attached' bones. 1a-w, Associated pieces lying in a natural position; 2,3, 'left' femur; 4a-j, 'right' and 'left' tibia/fibula (piece and counterpiece); 5a-e, 'right' foot/ankle (piece and counterpiece); 6, 7a,b, 8a-c, bone fragments; 9a-d, 10, 11, 12a-c, 13a,b, dromaeosaur tail pieces; A-HH, shims. See http://www.ctlab.geo.utexas.edu/pubs/ for the CT data set and further information.

bone, are usually separated from the other pieces by broad seams of grout, and preserve no evidence of a natural association with any piece in the first two groups. Shims were probably added at various times during assembly to secure the secondary bones into position around the bird skeleton and to make the slab more presentable.

In addition to the discrepancies revealed by the CT information, the tail of a nonvolant dromaeosaur also presented character conflicts with the ornithurine features of the bird skeleton and with the foot, which lacks the characteristic enlarged second claw of dromaeosaurids ${ }^{8}$. We found no evidence that the left femur, the split tibiae/fibulae, the split foot, or the tail belonged to the same individual or to the same species.

We conclude that Archaeoraptor represents two or more species and that it was assembled from at least two, and possibly five, separate specimens. Additional work in China verified that the tail is from an entirely different specimen, which has been described as a new species of dromaeosaur'.

Sadly, parts of at least two significant new specimens were combined in favour of the higher commercial value of the forgery, and both were nearly lost to science. Palaeontology was also badly damaged by the Piltdown forgery ${ }^{10}$ and the "lying stones" of Johann Beringer" ${ }^{11}$, and many fossils have been unwittingly or deliberately subjected to misleading reconstruction. Knowing the history of human handling can be critical to proper evaluation and scientific interpretation of specimens. Fortunately, a growing array of techniques can now be applied to forensic analysis of fossils.

Timothy Rowe ${ }^{\star}$, Richard A. Ketcham ${ }^{\star}$, Cambria Denison ${ }^{\star}$, Matthew Colbert ${ }^{\star}$, Xing Xu†, Philip J. Currieł

${ }^{*}$ High-Resolution X-ray Computed Tomography Facility, Department of Geological Sciences, C1100, The University of Texas at Austin, Austin, Texas 78712, USA

e-mail: rowe@mail.utexas.edu

$\dagger$ Institute of Vertebrate Paleontology and

Paleoanthropology, Academia Sinica, PO Box 643, Beijing 100044, PR China

$\ddagger$ Royal Tyrrell Museum of Paleontology, Box 7500, Drumheller, Alberta T0J OYO, Canada

1. Sloan, C. P. Natl Geogr. Mag. 196(5), 98-107 (1999).

2. Qiang, J., Currie, P. J., Norell, M. A. \& Shu-An, J. Nature 393, 753-761 (1998).

3. Xu, X., Wang, X.-L., Zhang, F.-C. \& Wu, X.-C. Nature 401, 262-265 (1999).

4. Dalton, R. Nature 403, 689-690 (2000).

5. Dalton, R. Nature 404, 696 (2000).

6. Holden, C. Science 288, 238-239 (2000).

7. Rowe, T., Kappelman, J., Carlson, W. D., Ketcham, R. A. \& Denison, C. Geotimes 42, 23-27 (1997).

8. Dingus, L. \& Rowe, T. The Mistaken Extinction - Dinosaur Evolution and the Origin of Birds (Freeman, New York, 1997). 9. Xu, X., Zhou, Z. \& Wang, X. Nature 408, 705-708 (2000). 10. Weiner, J. S. The Piltdown Forgery (Oxford University Press, London, 1955).

11. Jahn, M. E. \& Woolf, D. J. The Lying Stones of Dr. Johann Bartholomew Adam Berringe (University of California Press, Berkeley, 1963).

Supplementary information is available on Nature's website at http://www.nature.com or as a paper copy from the London editorial office of Nature. 